\title{
The need for quantitative PET in multicentre studies
}

\author{
Adriaan A. Lammertsma · Ronald Boellaard
}

Received: 15 June 2014 / Accepted: 25 July 2014/Published online: 16 August 2014

(C) Italian Association of Nuclear Medicine and Molecular Imaging 2014

Positron emission tomography (PET) was developed in the seventies of the last century with the first commercial scanner becoming available in 1978. Originally, this novel tomographic imaging technique was seen and used as a quantitative method for measuring human physiological and pathophysiological processes at regional level. The two most important characteristics of PET were its quantitative nature and its extremely high sensitivity, which allowed measurements down to picomolar level. Over the following 10-20 years the molecular processes that could be investigated using PET expanded rapidly, ranging from perfusion through metabolism, pre- and post-synaptic receptor density and affinity, neurotransmitter release, and enzyme activity, to drug delivery and uptake.

Given that PET, as mentioned, was characterised by two unique features (quantification and sensitivity), it is of interest to note that by the turn of the century one of them, sensitivity, was starting to be applied in clinical medicine, in particular its sensitivity to detect (distant) metastases using ${ }^{18}$ F-FDG. This led to a significant improvement in staging, which had direct consequences with regard to the choice of appropriate therapy (e.g. avoidance of futile surgery). As a result PET became the fastest growing medical technology, which was in sharp contrast to the situation in the mid-nineties, when the field (and the manufacturing industry) had been struggling to survive.

Color figures online at http://link.springer.com/article/10.1007/ s40336-014-0074-y

A. A. Lammertsma $(\bowtie) \cdot$ R. Boellaard

Department of Radiology and Nuclear Medicine, VU University

Medical Center, P.O. Box 1007, 1007 MB Amsterdam,

The Netherlands

e-mail: aa.lammertsma@vumc.nl
Clearly, to detect metastases (by finding hot spots), quantification was not necessary, as the purpose of a PET staging study is simply to establish whether they are there or not. In addition, it was convenient that scanning could be performed according to a protocol that is standard in clinical nuclear medicine practice, i.e. inject the tracer, wait for sufficient uptake in tissue and finally position and scan the patient. For ${ }^{18} \mathrm{~F}$-FDG, this waiting period is $1 \mathrm{~h}$ and, from the perspective of patient throughput, it is very convenient that the scanner is not occupied during this uptake period.

It is unfortunate and somewhat ironic, however, that one of the unique characteristics of PET (sensitivity) has indirectly been the reason why the other (quantification) is less recognised and indeed unknown to many clinicians who became familiar with PET through staging studies, in which quantification is not required. On the basis of its success in staging, however, PET is now seen as a very promising technique for monitoring and even predicting response to therapy. Indeed, ${ }^{18}$ F-FDG PET is already used as a surrogate endpoint in the development of new anticancer drugs. In addition, other more specific tracers are increasingly being used in drug development, both in oncology and in neurology. In this context, it is very tempting to implement the same methodology that is used in ${ }^{18}$ F-FDG staging studies, i.e. the application of a static or whole-body protocol at some time after injection of the tracer.

It should be noted that there are many aspects to quantification that need to be taken into account, i.e. scanner, physics and patient-related factors [1]. A prerequisite for quantification is that the scanner be equipped with accurate randoms, scatter and attenuation correction procedures. This may seem trivial, but it is well-known that the most recent scanners (i.e. PET/MRI) are associated with substantial errors in attenuation correction, even in the 
brain [2]. Similarly, there is a tendency to introduce novel (iterative) reconstruction algorithms because they generate nicer images, but without considering whether the reconstructed images are still accurate at both high and low radioactivity concentrations. From a physics point of view, the largest potential error is the partial volume effect due to the limited spatial resolution of PET. This can be a confounding factor in response monitoring studies [3], especially for smaller lesions that change in size as a result of therapy. Numerous correction methods have been proposed, many based on high-resolution anatomical images (CT or MRI). These methods are based on the assumption that anatomical and molecular (PET) volumes coincide, which is not the case in, for example, heterogeneous tumours. In addition, many of these methods provide inaccurate results or even fail in the presence of small lesions $(<1.5 \mathrm{~cm}$ diameter $)$ where partial volume effects are most pronounced. An alternative, less examined, technique that can be applied in dynamic studies is to incorporate a partial volume parameter in the tracer kinetic model [4]. Finally, there are several patient-related issues, the most notorious being patient movement. Progress is still being made in gating techniques to correct for cardiac and respiratory movements, although this remains difficult for kinetic studies. Both on-line and retrospective correction methods exist for dealing with the problem of patient movement during scanning, but it is essential to limit such movement as much as possible by paying attention to the patient during a scan.

As mentioned above, in routine clinical (nuclear medicine) practice, such as in staging using ${ }^{18} \mathrm{~F}-\mathrm{FDG}$, it is customary to inject the tracer, wait (in case of ${ }^{18} \mathrm{~F}-\mathrm{FDG}$ for $1 \mathrm{~h}$ ) and then acquire a static scan of tracer uptake. In general, increased uptake is associated with an increase in the physiological or molecular process being monitored. Net uptake at a certain time after injection, however, is the result of a complex interplay between delivery, uptake, retention and clearance. Therefore, increased uptake can also be due to increased availability, i.e. either increased plasma concentration or increased flow, or decreased clearance. From a single static scan, it is impossible to separate the various components that contribute to the total signal, such as specific binding, non-specific binding and free tracer in tissue. This problem is clearly illustrated in Fig. 1, where images of total uptake (left) are compared with those of specific binding (right) before and after administration of a pharmacological dose of an antagonist. It is obvious that it is impossible to estimate the large change in specific binding $(>95 \%)$ from the images of total uptake, where the change in total signal is much smaller.

PET studies in drug development can roughly be divided into three categories. First, labelled drugs can be used to assess whether a drug targets the lesion, e.g. a tumour.



Fig. 1 Uptake of the NK1 receptor ligand ${ }^{11} \mathrm{C}-\mathrm{R} 116301$ in a normal volunteer with (left) total uptake images (60-90 min after injection) and (right) parametric images of non-displaceable binding potential $\left(\mathrm{BP}_{\mathrm{ND}}\right)$, representing specific signal only. The top row represents images at baseline, the bottom row corresponding images after a blocking dose of aprepitant. Quantitative analysis of the $\mathrm{BP}_{\mathrm{ND}}$ images showed that aprepitant occupied $97 \%$ of the NK1 receptors, a proportion substantially higher than expected on basis of the total uptake images (color figure online)

Many qualitative studies are being performed, most notably ones with labelled monoclonal antibodies. In the latter, a dose of cold antibody is often given to saturate the liver, thereby precluding proper quantification as the saturating dose will also occupy some of the binding sites in the lesion itself. This is acceptable as long as the only purpose of such a study is to assess whether a certain target is expressed in the lesion, but even then subtle differences in target expression may be missed. More importantly, when PET is used to predict response, quantification is essential especially when response is related to local tissue concentrations [5]. In addition, non-specific uptake may obscure the specific signal. For example, it has been shown that quantitative studies (measuring volume of distribution) using carbon-11-labelled erlotinib provide complete separation between lung cancer patients with mutated (responders) and wild-type (non-responders) EGFR, whilst substantial overlap is seen when using semi-quantitative SUV images [6].

In the second category of PET studies performed in drug development it is not the labelled drug itself that is used, but rather an established and validated tracer of the binding site that is targeted by the drug. The classical example of 
this approach is the determination of D2 receptor occupancy by neuroleptics. Quantification is necessary as it is generally accepted that the therapeutic range is $65-85 \%$ receptor occupancy [7]. More recently, however, the same approach has been used for imaging and measuring amyloid load to assess the efficacy of amyloid-modifying drugs. It is of interest to note that all major trials have used a semi-quantitative approach, namely SUVr (target area SUV normalised to cerebellum SUV). Although not validated, this approach has been defended on the basis of the following arguments: (a) a simpler scanning protocol allows the participation of less experienced PET centres, thereby increasing patient accrual, (b) a simpler scanning protocol means lower PET scanning costs, and (c) by normalising to an internal standard (cerebellum) any confounding effect will be largely compensated for. The first argument may be true, but by including less experienced centres not only will more patients be included, but also more spread in the data, meaning that the improvement in statistical power may be marginal (and obtained at much higher costs because of the larger number of subjects that need to be scanned). The second argument is true, but essentially irrelevant within the context of drug development costs. In addition, more accurate fully quantitative studies may require the enrolment of fewer patients. Finally, the third and scientifically most important argument must be considered wrong, at least until it is validated in independent studies. For example, SUVr has been used to evaluate amyloid targeting therapy, but in a recent study [8] it was shown that in a time span of two to 3 years, ${ }^{11} \mathrm{C}$ PIB SUVr actually decreased in Alzheimer's disease (AD) patients who did not receive anti-amyloid therapy. By contrast, non-displaceable binding potential, i.e. the specific amyloid-related signal, remained unchanged. Using simulation studies, it was shown that the reduction in $\mathrm{SUVr}$ was likely due to a reduction in cerebral blood flow, which is known to occur in progressive AD. Clearly, SUVr should not be used to evaluate (probably subtle) changes due to amyloid targeting therapy (note that delivery, too, may be affected by a different body distribution as a result of the therapy), as it could even provide misleading information.

The third and most common application of PET studies in drug development is for monitoring (functional) response (early) during therapy, most notably through the measurement of glucose metabolism following chemotherapy. In this case, there is a large body of evidence that a simple ${ }^{18}$ F-FDG SUV measurement is a good surrogate marker of glucose metabolism (MRglu) as obtained from full kinetic studies, at least in classical chemotherapy. Nevertheless, for new drugs, maintenance of this relationship should be validated. Especially for novel biologicals, the body distribution of ${ }^{18}$ F-FDG may change, affecting their delivery to the tumour (e.g. due to an inflammatory response elsewhere in the body). This may lead to a change in the relationship between SUV and MRglu after therapy [9-11]. Consequently, the relationship between SUV and MRglu in pre- and post-therapy scans could be different, potentially leading to over- or underestimation of response based on SUV. For this reason, both EORTC [12] and NCI [13] recommend that in a multicentre trial at least one group should investigate this relationship.

Multicentre PET studies are primarily undertaken with ${ }^{18} \mathrm{~F}$-FDG, but in principle they may be needed for other tracers as well. A recent example is the assessment of amyloid load using a variety of amyloid tracers. Even for more complicated scanning protocols using novel tracers, multicentre studies may be needed to increase the statistical power, especially when patient enrolment in a single centre would be too slow ("rare" diseases). In such cases, it is important that scanning conditions between centres are standardised, especially for absolute measurements relative to injected dose (i.e. SUV). Reference tissue methods (kinetic reference tissue models or SUVr) have an advantage in that an internal (calibration) standard is used. Harmonisation demands attention to several study aspects: patient preparation (e.g. dietary conditions), scanning protocol (e.g. timing of scans), reconstruction protocol (e.g. harmonising signal-to-noise ratios), image analysis (e.g. region of interest definition) and actual data analysis methods.

Harmonisation is a key issue when using PET to quantify imaging biomarkers in multicentre studies. In 2001, the 'Biomarkers definition working group' defined a biomarker as: "a characteristic that is objectively measured and evaluated as an indicator of normal biologic processes, pathogenic processes, or pharmacologic responses to a therapeutic intervention" [14]. Biomarkers should have high feasibility, precision and accuracy in any medical centre participating in a multicentre trial. In other words, they should show high repeatability and reproducibility in order to be used as validated and (FDA or EMA) approved surrogate endpoints. Repeatability is a measure of the uncertainty of obtaining the same result when scanning the same patient under the same conditions on the same system. Therefore, it is mainly indicative of the biological component of the overall variability or uncertainty. This uncertainty can usually be minimised by standardising or harmonising patient preparation procedures and by using exactly the same scanning protocol. Reproducibility is a measure of the variability in or uncertainty of obtaining the same result in the same patient when being studied on different PET systems in different institutes. In other words, reproducibility also includes uncertainties originating from the use of different PET systems, reconstruction methods, and imaging and data analysis procedures (between institutes). High reproducibility can be obtained 
only when all technical factors, PET image characteristics (such as noise, contrast, recovery, etc.) and data analysis procedures are strictly harmonised. An often overlooked issue is that these factors should not be addressed in isolation. The final quantitative readout of any PET study depends on the combination of all these factors, i.e. patient preparation, technical factors, imaging characteristics and data analysis methods. For example, for oncological ${ }^{18} \mathrm{~F}$ FDG PET studies about 12 different factors have been identified and characterised, all having an uncertainty of approximately 10-15\%. The cumulative error when these factors are not strictly harmonised could easily lead to a two fold difference in observed SUV between imaging systems and sites [15, 16].

Although significant progress has been made, detailed aspects of the harmonisation of ${ }^{18} \mathrm{~F}$-FDG PET studies are still being discussed some 20 years after it was recognised that harmonisation is important in order to combine data from different institutes (i.e. in multicentre trials). Clearly, the level of harmonisation also depends on the size of the expected signal in relation to the reproducibility of the measurements. In many cases, the potential therapyinduced bias in signal will be smaller than the change in ${ }^{18}$ F-FDG uptake following successful chemotherapy. In order not to waste time and resources on futile trials, it is important (a) that simplified protocols and measures are used only after they have been validated against fully quantitative analyses, and (b) that lessons learned from ${ }^{18} \mathrm{~F}$ FDG harmonisation are adhered to and generalised to other tracers. In such conditions, PET can be a valuable, sensitive and quantitative imaging biomarker in drug development studies.

Conflict of interest A. A. Lammertsma and R. Boellaard declare no conflict of interest.

\section{References}

1. Zaidi H, Alavi A (2014) Trends in PET quantification: opportunities and challenges. Clin Transl Imaging 2:183-185. doi:10. 1007/s40336-014-0065-z

2. Dickson JC, O'Meara C, Barnes A (2014) A comparison of CTand MR-based attenuation correction in neurological PET. Eur J Nucl Med Mol Imaging 41:1176-1189

3. Hess S, Blomberg BA, Rakheja R, Friedman K, Kwee TC, Hoilund-Carlsen PF, Alavi A (2014) A brief overview of novel approaches to FDG PET imaging and quantification. Clin Transl Imaging 2:187-198. doi:10.1007/s40336-014-0062-2

4. Iida H, Kanno I, Takahashi A, Miura S, Murakami M, Takahoshi K, Ono Y, Shishido F, Inugami A, Tomura N, Higano S, Fujita H, Sasaki H, Nakamichi H, Mizusawa S, Kondo Y, Uemura K (1988) Measurement of absolute myocardial blood flow with
H2150 and dynamic positron emission tomography: strategy for quantification in relation to the partial volume effect. Circulation 78:104-115

5. Van der Veldt AAM, Lubberink M, Mathijssen RHJ, Loos WJ, Herder GJM, Greuter HN, Comans EFI, Rutten HB, Erikson J, Windhorst AD, Hendrikse NH, Postmus PE, Smit EF, Lammertsma AA (2013) Toward prediction of efficacy of chemotherapy: a proof of concept study in lung cancer patients using $\left[{ }^{11} \mathrm{C}\right]$ docetaxel and positron emission tomography. Clin Canc Res 19:4163-4173

6. Bahce I, Smit EF, Lubberink M, van der Veldt AAM, Yaqub M, Windhorst AD, Schuit RC, Thunnissen E, Heideman DAM, Postmus PE, Lammertsma AA, Hendrikse NH (2013) Development of $\left[{ }^{11} \mathrm{C}\right]$ erlotinib positron emission tomography for in vivo evaluation of EGF receptor mutational status. Clin Cancer Res 19:183-193

7. Farde L, Wiesel FA, Halldin C, Sedvall G (1988) Central D2dopamine receptor occupancy in schizophrenic patients treated with antipsychotic drugs. Arch Gen Psychiatry 45:71-76

8. Van Berckel BNM, Ossenkoppele R, Tolboom N, Yaqub M, Foster-Dingley JC, Windhorst AD, Scheltens P, Lammertsma AA, Boellaard R (2013) Longitudinal amyloid imaging using ${ }^{11} \mathrm{C}-\mathrm{PiB}$ : methodologic considerations. J Nucl Med 54:1570-1576

9. Freedman NM, Sundaram SK, Kurdziel K, Carrasquillo JA, Whatley M, Carson JM, Sellers D, Libutti SK, Yang JC, Bacharach SL (2003) Comparison of SUV and Patlak slope for monitoring of cancer therapy using serial PET scans. Eur J Nucl Med Mol Imaging 30:46-53

10. Doot RK, Dunnwald LK, Schubert EK, Muzi M, Peterson LM, Kinahan PE, Kurland BF, Mankoff DA (2007) Dynamic and static approaches to quantifying ${ }^{18}$ F-FDG uptake for measuring cancer response to therapy, including the effect of granulocyte CSF. J Nucl Med 48:920-925

11. Cheebsumon P, Velasquez LM, Hoekstra CJ, Hayes W, Kloet RW, Hoetjes NJ, Smit EF, Hoekstra OS, Lammertsma AA, Boellaard R (2011) Measuring response to therapy using FDG PET: semi-quantitative and full kinetic analysis. Eur J Nucl Med Mol Imaging 38:832-842

12. Young H, Baum R, Cremerius U, Herholz K, Hoekstra O, Lammertsma AA, Pruim J, Price P (1999) Measurement of clinical and subclinical tumour response using $\left[{ }^{18} \mathrm{~F}\right]$-fluorodeoxyglucose and positron emission tomography: review and 1999 EORTC recommendations. Eur J Cancer 35:1773-1782

13. Shankar LK, Hoffman JM, Bacharach S, Graham MM, Karp J, Lammertsma AA, Larson S, Mankoff DA, Siegel BA, Van den Abbeele A, Yap J, Sullivan D (2006) Consensus recommendations for the use of ${ }^{18}$ F-FDG PET as an indicator of therapeutic response in patients in National Cancer Institute trials. J Nucl Med 47:1059-1066

14. Biomarkers Definitions Working Group (2001) Biomarkers and surrogate endpoints: preferred definitions and conceptual framework. Clin Pharmacol Ther 69:89-95

15. Boellaard R (2009) Standards for PET image acquisition and quantitative data analysis. J Nucl Med 50(Suppl 1):11S-20S

16. Lasnon C, Desmonts C, Quak E, Gervais R, Do P, Dubos-Arvis C, Aide N (2013) Harmonizing SUVs in multicentre trials when using different generation PET systems: prospective validation in non-small cell lung cancer patients. Eur J Nucl Med Mol Imaging 40:985-996 